\title{
Des méthodes combinées pour une analyse communicationnelle d'une liste de discussion professionnelle
}

Combining Methods for a Communicational Analysis of a Professional Discussion List

Florence Thiault

\section{(2) OpenEdition}

Journals

Édition électronique

URL : http://journals.openedition.org/edc/1096

DOl : 10.4000/edc. 1096

ISSN : 2101-0366

Éditeur

Université Lille-3

Édition imprimée

Date de publication : 1 décembre 2009

Pagination : 203-216

ISSN : 1270-6841

Référence électronique

Florence Thiault, «Des méthodes combinées pour une analyse communicationnelle d'une liste de discussion professionnelle », Études de communication [En ligne], 33 | 2009, mis en ligne le 01 décembre 2011, consulté le 19 avril 2019. URL : http://journals.openedition.org/edc/1096 ; DOI : 10.4000/edc. 1096

Ce document a été généré automatiquement le 19 avril 2019

(c) Tous droits réservés 


\section{Des méthodes combinées pour une analyse communicationnelle d'une liste de discussion professionnelle}

Combining Methods for a Communicational Analysis of a Professional Discussion List

Florence Thiault

1 Nous nous intéressons dans nos travaux au fonctionnement d'une communauté professionnelle en information-documentation. A partir de l'étude des archives, nous analysons les échanges de documentalistes sur une liste de discussion sur la documentation et internet dans l'établissement scolaire (cdidoc). La communication électronique permet-elle l'expression de nouvelles formes d'échange, de production de savoir et d'information au sein de cette communauté professionnelle? La liste de diffusion est appréhendée à la fois comme un espace récapitulatif d'une situation professionnelle et comme un espace qui permet de percevoir les nouveaux questionnements d'une profession. Comment la communauté utilise ce qui est élaboré collectivement? Les échanges dans la communauté des membres d'une liste de discussion professionnelle peuvent-ils constituer un corpus de connaissances source de nouveaux savoirs pour les acteurs? Le regard communicationnel que nous portons sur l'objet « communauté de pratiques en ligne » suppose d'observer et d'analyser la dynamique des relations, les jeux et stratégies d'acteurs, les pratiques sociales, les échanges, les représentations. Trois grandes catégories d'acteurs sont présents sur cdidoc, une minorité active qui correspond aux producteurs réguliers de ressources, des contributeurs occasionnels qui interviennent sur des questions de résolutions de problèmes et une majorité silencieuse d'abonnés. Etudier le fonctionnement de cette liste de discussion destinée aux documentalistes est un moyen d'approcher sous un certain angle les modes et degré de structuration de ce groupe professionnel. Les dimensions exemplaires du terrain analysé nous renvoient au fonctionnement d'autres communautés professionnelles à faible légitimation, en situation de construction de savoirs communs. 
2 Cette contribution vise à décrire les choix méthodologiques qui ont été effectués afin de construire notre objet d'étude. Pour établir notre propos, nous présenterons le dispositif méthodologique tel que nous l'avons construit à partir du croisement de différentes méthodes, nous exposerons dans un deuxième temps notre cadre théorique d'analyse, nous terminerons par l'analyse des résultats d'une étude exploratoire sur les activités professionnelles dominantes au sein du corpus.

\section{La construction d'un dispositif méthodologique composite}

\section{Une approche ethnologique à visée anthropologique}

3 Le terrain est le point de départ de notre problématique dans une approche de type constructiviste. La familiarité avec le terrain, construite au fil de l'exercice professionnel apporte des informations, des repères conséquents et contribue à faire mûrir un questionnement permettant de conduire une recherche. La démarche choisie prend en compte notre degré d'identification à l'endogroupe avec ses limites et ses avantages. Notre approche ethnographique s'appuie sur l'analyse des traces écrites, des échanges, des codes et rituels dans la communauté particulière des membres de la liste cdidoc. Pour analyser cette communauté, nous avons choisi de croiser différentes méthodes dans une logique de complémentarité, afin de constituer des regards distincts sur notre objet de recherche et de juxtaposer les angles d'observation. Nous avons adopté une approche qualitative par la méthode anthropologique ce qui implique pour notre étude une observation participante sur le terrain d'enquête et la constitution d'un corpus de documents écrits. Yves Winkin décrit dans "Anthropologie de la communication » cette méthode qui permet d'organiser la façon de rassembler les matériaux et la manière de les observer, puis de définir le mode d'analyse qui leur est adapté : « Tout d'abord, il s'agit de dégager par observation participante les cadres de perception et d'organisation par lesquels certains phénomènes naturels et sociaux sont tenus, dans un groupe social donné, pour des événements ou des actes de communication" (Winkin, 1996, 85). L'objectif principal est alors d'étudier les pratiques des acteurs en situation, et d'approcher la culture de chaque organisation pour comprendre dans quel contexte sont produits les discours émanant de ces organisations. Notre dessein est de chercher des archétypes, de parvenir à repérer des régularités qui soient généralisables. En ce sens l'aller et retour entre la théorie et la pratique est permanent. Ce travail d'enquête nous a permis de procéder à un recueil de «traces » écrites prélevées sur le terrain, témoignant d'interactions entre les acteurs. Notre intention est de faire apparaître la complexité observée et de penser l'hybridité socio-technique du dispositif.

\section{Une analyse communicationnelle du corpus}

Cette approche ethnographique est complétée par une analyse communicationnelle longitudinale des contenus des messages. Cette dernière permet d'observer l'apparition de nouveaux questionnements (lors de la mise en place de récents enseignements, par exemple l'éducation à l'environnement pour un développement durable en 2004), la continuité et la récurrence ou non de certains sujets (la venue d'un auteur dans la classe), la manière dont se construit un rapport aux savoirs (l'utilisation du thésaurus dans le 
cadre d'une recherche documentaire informatisée). L'analyse des discussions porte sur une double qualification: la formulation et la reconnaissance de savoirs partagés; la formation et la transformation du jugement. Qu'ils soient présentés sous formes de conseils, de mises au point, d'information ou de questions ouvertes, la liste de discussion met en scène et régule un (des) rapport(s) aux savoirs. Au-delà des modalités de prise de paroles et des prises de risque résultant de l'exposition publique, la liste de discussion configure les situations problèmes, les solutions et les bonnes pratiques. Se faisant, la communauté incorpore dans le « cognitif » des aspirations professionnelles et sociales, au point où les savoirs partagés deviennent un support de légitimation de la liste et plus largement de la profession. La reconnaissance d'un savoir résulte d'une dynamique complexe et politique dans laquelle se jouent des prétentions à camper un monde commun, activité qui présuppose une capacité de chacun à examiner ou proposer du possible. La communauté est un ensemble d'individualités, chacun portant des attentes, des idées et des jugements. Nicole d'Alméida $(2007,11)$ définit le jugement comme « une intervention sur le monde engageant une activité spécifique qui n'est ni calculatrice, ni désintéressée [...]. Dans le jugement, l'individu se confronte au monde et lui donne une intelligibilité, un sens [...]. Le jugement relève du sens commun, du sens de la communauté. Juger en tant que membre d'une communauté, c'est entrer en société ». Le processus de qualification des personnes et des savoirs partagés constitue un inclassable travail de tissage d'un récit venant mettre en ordre et en sens une profession.

\section{Les cadres théoriques d'analyse}

\section{L'apport de l'analyse discursive}

5 Nous portons dans notre travail de recherche une attention particulière aux productions discursives des acteurs afin d'étudier l'évolution de leurs discours, d'analyser la dialectique qui se joue entre convergence et divergence et afin d'y rechercher les traces de la construction d'un langage commun, condition de toute démarche collective. Nous avons associé pour l'étude de notre corpus l'analyse de contenu et l'analyse de discours selon la définition qu'en donne Simone Bonnafous.

«L'analyse du discours s'intéresse à la forme du message médiatique, politique, public, gouvernemental, organisationnel, etc., en rapport avec son lieu social de production (le contexte historique et sociologique, le média, le parti politique, le gouvernement, l'entreprise, etc.). C'est donc une démarche fondée sur la linguistique, mais qui fait le lien entre le discours et le social, entre le verbal et l'institutionnel, entre les mots, les figures, les arguments et ceux qui les énoncent, dans leur dimension aussi bien individuelle que collective » $(2006,213)$.

6 Nous pouvons dégager plusieurs niveaux dans notre analyse de corpus. Lors d'une première phase, l'analyse thématique et lexicale d'un échantillon de messages nous permet de repérer les convergences/divergences présentes dans les discours (thèmes et vocabulaire communs). L'analyse thématique recense les tendances des différents discours, son but est d'aboutir à une grille catégorielle indiquant la fréquence d'apparition des thèmes. L'analyse lexicale quant à elle, s'attache à étudier le vocabulaire, les caractéristiques grammaticales, les qualificatifs employés dans les discours des uns et des autres. Cette analyse d'un échantillon est complétée par une méthode de statistique lexicométrique sur l'ensemble du corpus avec le logiciel Wordmapper (de Grimmersoft) pour découvrir des évolutions, des régularités et des ruptures, qu'une lecture cursive ne 
permet pas de déceler. Le retour au contexte permet ensuite des analyses plus fines. Les archives de la liste de discussion font l'objet d'une étude de contenu, plus précisément une analyse catégorielle, pour dégager les thèmes les plus pertinents que nous étudions individuellement. Une analyse manuelle de chaque message retenu dans un échantillon représentatif est effectuée à partir d'une grille d'analyse spécialement créée dans le cadre de ce projet. Cette grille d'observation constitue un métalangage sur la situation. Elle enregistre dans un nombre restreint de catégories (items) des comportements délimités comme frappants permettant de voir le degré, la fréquence de leur apparition dans une situation particulière ou d'en comparer plusieurs.

\section{La rhétorique des acteurs}

7 Une autre dimension à examiner concerne l'analyse de la rhétorique des acteurs. Celle-ci se décline notamment par l'ethos ou présentation de soi des acteurs qui nous paraît très intéressant à étudier dans l'idée de dégager un « ethos professionnel » pour observer son évolution, révélatrice de l'évolution de l'identité des acteurs. L'étude des éléments de la signature des messages (anonymat, lieu d'exercice, fonction revendiquée...) nous interroge sur le choix de l'appellation du professionnel en exercice (enseignant documentaliste, professeur documentaliste, documentaliste, prof doc, e-doc). Les logiques de présentation de soi sont étroitement liées avec les stratégies argumentatives déployées par les acteurs, dans un but de persuasion, ainsi certains contributeurs précisent leurs fonctions de formateurs pour légitimer leurs expertises. Ce qui est en jeu avec l'ethos, c'est l'importance de la représentation de soi et de l'autre dans l'interlocution. Une autre notion clé de la rhétorique nous paraît être essentielle pour notre analyse, la notion de praxis. La communication porte sur les activités précises des acteurs. Les logiques de médiation rejoignent alors les logiques de discours sur soi. Il s'agit de présenter ce que l'on fait, ou ce que l'on va faire. Le dire est dès lors inséparable du faire, de la praxis. Ainsi, derrière les discours sur le métier, il y a des acteurs, des interactions, des inscriptions sociales et institutionnelles.

\section{La voix du tiers}

8 A la suite d'auteurs comme Patrick Charaudeau et Rosa Montes qui mettent en avant une conception triadique de la communication, nous postulons la présence d'un tiers comme figure jouant le rôle de garant dans le partage de pratiques. Patrick Charaudeau distingue trois lieux de problématisation du tiers: "l'espace situationnel de la communication", "l'espace discursif de l'énonciation », « l'espace interdiscursif où circulent les discours ». Ce dernier lieu, l'espace interdiscursif, défini comme «lieu d'une sémantisation des systèmes de valeurs, où le tiers peut être considéré comme un «méta-énonciateur » qui produirait des discours de vérité servant de référence à tout nouvel énonciateur », retient particulièrement notre attention. Ce répondant qui donne du sens, ce surdestinataire comme le dit Bakhtine est celui qui sous-tend la circulation de discours toujours contextualisés, porteurs de systèmes de valeurs à référer à des systèmes de pensée constitués en " imaginaires socio-discursifs ». La notion d' "imaginaire socio-discursif " est essentielle en ce qu'elle façonne les représentations des différents acteurs. Au sein de notre corpus constitué de discours circulant, nous recherchons donc les traces et manifestations de ce "méta-énonciateur" dans notre corpus. Les abonnés actifs construisent leurs discours à travers les destinataires et lecteurs supposés. La 
construction identitaire s'affirme ainsi autour de l'exposition des opinions. Pour Charaudeau, la position du tiers est fondamentale dans le discours, le regard de l'autre entrainerait des différences dans les objets discutés. Les participants de la liste de discussion partagent des savoirs de connaissance et de croyance, savoirs qui circulent dans le groupe auquel ils appartiennent et qui sont mobilisés dans un jeu d'interdiscursivité. La référence au tiers est présente dans l'exemple suivant, le surdestinataire est identifié par "il" dans les expressions "il faut, il est toujours ...".

«Message 1:

Sujet : bcdi : désherbage périodiques et exemplaires

Bonjour tous! depuis mon arrivée en septembre dernier dans mon établissement, j'ai effectué un grand désherbage des périodiques. J'ai supprimé la fiche documentnotice de chaque périodique désherbé et conservé la fiche exemplaire avec statut $=$ mis au pilon. Mais je me demande à présent si il faut ou non conservé la fiche exemplaire? Ces 1700 exemplaires mis au pilon ne surchargent-ils pas la base de données? Merci

Message 2

Ces exemplaires sont la mémoire du fond, il faut donc les conserver.... ils ne sont pas très lourds et ne gênent en rien la recherche, donc pas de soucis.

Message 3

Bonjour, je ne crois pas qu'il faille supprimer ces exemplaires... Il est toujours recommandé de les conserver car il s'agit de documents achetés sur le budget de l'éducation nationale... Les 1700 exemplaires ne surchargent pas la base... Dans le lycée où j'étais, la base contenait plus de 10000 exemplaires... Bonne journée »

Les occurrences de "il faut" renvoient à une norme professionnelle non citée. Le tiers apparait comme une instance de jugement, un arbitre social de la norme, chargé de décider de l'acceptabilité sociale des comportements des acteurs.

\section{Communauté de pratique et agencement des savoirs}

Les technologies de l'information et de la communication ont transformé le métier d'enseignant documentaliste et ne cesse de le faire évoluer. Elles ont permis l'émergence d'une communauté de pratique qui s'appuie sur une reconnaissance endogène des savoirs informationnels et documentaires. Qu'est-ce qu'une communauté de pratique ? D'après Wenger, il s'agit d'un groupe de personnes qui ne travaillent pas nécessairement ensemble mais qui interagissent pour échanger de l'information, des points de vue et des conseils, solutionner des problèmes et approfondir des connaissances dans un domaine de pratiques pour lequel elles partagent un intérêt (Wenger, 1998). Une communauté de pratique désigne donc des personnes liées entre elles par le partage de pratiques communes, ce qui induit le développement d'un langage commun et d'une compréhension partagée de leur environnement professionnel. Pour Wenger, une communauté de pratique peut se décrire à partir de trois éléments articulés: un engagement mutuel, une entreprise commune, le répertoire partagé. L'engagement mutuel c'est l'idée que dans une communauté de pratique, l'individu va pouvoir être identifié par les pairs, et en même temps qu'il va travailler à l'amélioration d'une identité individuelle et collective. L'entreprise commune renvoie au fait que les communautés de pratique se constituent généralement autour de la notion de résolution de problèmes dans une dimension pragmatique. En ce qui concerne le répertoire partagé la communauté passe d'une conscience pratique à une conscience discursive, qui se confirme dans un processus de construction de ressources symboliques. Ce qui nous intéresse dans la constitution de ce répertoire partagé, c'est les questions de doxa et/ou 
de doctrine d'action. En effet, la communauté étudiée des professeurs documentalistes est dans un processus de construction de savoirs opératoires qui deviennent des savoirs constituants du collectif (Sarfati, 2002). A partir de l'analyse d'une granulation fine de l'information dans cette communauté de pratique, nous pouvons essayer de voir comment s'opère les transitions. Il nous est possible de repérer par une analyse longitudinale l'apparition d'enjeux, leurs mises en cause, leurs constructions dans le temps et comment ils deviennent des incantations ou des réactions. Les sujets abordés dans la liste discussion cdidoc concernent le champ professionnel de la documentation scolaire. Nous trouvons plusieurs types d'informations circulant sur la liste. Les questions représentent les besoins en connaissances, les réponses correspondant aux connaissances proposées à la mutualisation. La nature des questions varie mais nous pouvons distinguer deux sortes principales: les demandes de retour d'expérience et les demandes d'information. L'objectif des membres est d'améliorer les conditions de l'exercice de leur profession au quotidien par le partage, l'entraide et les processus d'apprentissage et enseignement mutuels. La participation à l'activité de la communauté de pratique virtuelle permet aux membres de prendre conscience du fait qu'ils possèdent des préoccupations et des intérêts communs. A la longue, la communauté acquiert une histoire qui peut être partagée avec de nouveaux membres, à travers les objets que ses participants ont créés (les échanges sont consignés dans les archives) ou les actions qu'ils ont menées. Comme l'indique Elisabeth Kolmayer, « la liste est bien un lieu d'expression de besoins de connaissances pour l'action, insérées dans l'activité professionnelle ». C'est l'un des traits caractéristiques d'une communauté.

\section{Les activités professionnelles dominantes}

\section{Méthodologie de l'étude exploratoire}

11 C'est à partir du discours des individus engagés dans des pratiques professionnelles que l'analyse s'effectue. La recherche qualitative de type ethnographique constitue un outil des plus appropriés pour mettre en lumière la complexité du processus de construction identitaire et les pratiques langagières qui s'y rattachent. Après examen d'un échantillon du matériau empirique (2637 messages archivés de janvier 2000 à décembre 2006), nous avons réalisé une première analyse thématique des échanges sur la liste en nous appuyant sur l'examen des mots-clés utilisés le plus fréquemment par l'indexeur ( $1 \%$ et plus). Les opérations intellectuelles sollicitées sont le repérage, le regroupement et l'étude des thèmes abordés dans le corpus par une démarche discursive de reformulation et d'explicitation. Chaque message constitue une notice structurée autour de différents champs (auteur, titre, date de publication, catégorie de question, mots clés libres ...). La production d'un métalangage de descripteurs nous aide à identifier les thématiques principales de la liste. Pour élaborer une catégorisation thématique du corpus, nous nous sommes appuyés sur l'Euroréférentiel I\&D publié par l'ADBS (association des professionnels de l'information et de la documentation) en 2004. L'objectif de l'ADBS est d'aider les professionnels à définir leur emploi, à caractériser leurs savoirs et savoir-faire et leur permettre d'évaluer leurs compétences et qualifications. Cette démarche de qualification s'appuie sur le modèle de la compétence (Zarifian, 2004) par l'écriture de l'activité comme élément de la professionnalisation. Ce référentiel est constitué de cinq 
groupes de compétences (information, technologies, communication, management, autres savoirs) associés à des domaines de compétences spécifiques pour chaque groupe.

\section{Terminologie et exercice du métier}

12 Le groupe central au cœur des métiers de l'I\&D est celui de l'information. Dans notre corpus, un noyau est constitué autour du domaine de la «compréhension du monde professionnel ». Les mots clés associés correspondent à des messages relatifs au mode de recrutement des enseignants documentalistes, aux problèmes liés au statut des personnels, à des questions sur le positionnement de la profession par rapport au groupe professionnel des enseignants et à une définition du périmètre des tâches constitutives de l'identité de la profession. Une grande partie de ce thème concerne la recherche de poste de contractuel ou des demandes d'informations sur les mutations et postes disponibles. Dans ce groupe, nous pouvons identifier toutes les questions statutaires vives de la profession sur les conditions de service (circulaire de mission, inspection, agrégation, salaire, heures supplémentaires, grève, vacances) et une interrogation sur des missions facultatives (professeur principal, conseil de classe) et sur l'avenir de la profession. Ces questions donnent lieu à des échanges vifs sur le métier comme dans l'exemple suivant qui reprend la plupart des revendications de la profession.

«DEBAT : Pb reconnaissance : Que faire ?: Résister, nom d'un chien! Nous avons passé un Capes et avons donc été "embauché(e)s" à ce titre par l'Education Nationale. Nous avons des missions, elles sont écrites dans une circulaire! Personne ne songerait à demander au prof d'anglais de faire des tâches de secrétariat ni au Principal d'aller aider en cuisine, ni aux surveillants de faire le ménage. Alors stop aux abus, faites votre boulot et uniquement lui, rédigez un projet CDI, mettez sous le nez de votre principal la circulaire de 86 et les protocoles d'inspection [...]. »

13 Cette rhétorique du métier nous renvoie aux problèmes de faible légitimation de la fonction de professeur documentaliste dans l'établissement scolaire et à la difficulté à faire reconnaître son utilité sociale. Cette légitimité est mise en jeu dans les transformations de l'exercice du métier : concurrence avec des personnels non formés, des savoirs professionnels pratiques plus qu'académiques, une autonomie limitée du professionnel. Le rôle principal du documentaliste est de mettre à disposition des enseignants et des élèves des documents répondant aux besoins pédagogiques ou culturels. Autour de cette activité principale de collecte, gestion et exploitation de documents et d'information sont présents plusieurs sous-thèmes sur la liste, dont un central sur "l'enrichissement des collections et des fonds». Les participants sollicitent des avis et conseils sur des sélections de ressources à acquérir pour approvisionner le secteur fictions du fonds documentaire, en littérature jeunesse en collège et en littérature en lycée et sur le choix de supports multimédias (cédérom, DVD).

«QUEST : Biblio fantastique $4^{\text {ème }}:[. .$.$] Un projet de défi lecture niveau 4ème a été$ monté en début d'année sur le thème du policier. A la rentrée, changement d'équipe enseignants et du doc ! ! La prof de français coordonnatrice qui se retrouve avec ce projet vient de changer de thème : le fantastique (qui correspond plus au niveau 4ème)... Donc si vous avez des propositions de romans fantastiques de différents niveaux de lecture et quelques titres de $\mathrm{BD}$, je suis partante, merci d'avance ».

14 Ces enrichissements de collection se font la plupart du temps dans le cadre de projet pédagogique spécifique, l'objectif $\mathrm{du}$ documentaliste est l'adaptation $\mathrm{du}$ fonds documentaire aux besoins de la communauté scolaire. Par ailleurs, de nombreux 
messages portent sur des questions de cotation des documents, thème qui fait référence à « l'analyse et représentation de l'information » compétence qui vise à identifier et décrire le contenu d'une ressource documentaire par l'indexation.

«TECHDOC : Cotation Carrés classiques: Bonjour, pourquoi ne pas coter les contes avec $\mathrm{C}$ et les nouvelles avec $\mathrm{R}$ ou $\mathrm{N}$ ? Ca te simplifierait bien la tâche et celle des élèves pour les retrouver. Sinon, les anthologies sont à classer en 840.8 ou 808.83 , puis te reporter à la table $3 \mathrm{C}$ de la Dewey. Bon courage ! »

Les abonnés interrogent les co-listiers sur le choix d'un indice de classification DEWEY pour intégrer le document dans la collection en libre accès. Un autre thème complémentaire concerne la "gestion des collections et des fonds » avec deux problèmes principalement évoqués, la gestion des périodiques (choix, conservation, gestion d'abonnement) et la question polémique de la gestion des manuels scolaires par le documentaliste. Quant au groupe Technologie, thème très présent dans les échanges sur les réseaux informatiques, il comprend toutes les questions se référant aux « technologies de l'information et de la communication » qui correspondent pour les documentalistes à la gestion, la maintenance, la surveillance du parc informatique dont ils ont la responsabilité. Dans le domaine de la «conception informatique de systèmes d'information documentaire ", les messages de notre corpus renvoient à l'utilisation du logiciel documentaire BCDI (paramétrage, version, gestion des prêts, inventaire...) et à des questions d'informatisation, de mutualisation des données et de formation des usagers.

Questions initiale : «QUEST : Pour les experts de BCDI : Bonjour, j'ai besoin de votre aide ! Je fais depuis un bon bout de temps le dépouillement de Mon quotidien, et je m'aperçois que j'ai fait une bête erreur. Pour faire court, je voudrais faire un changement par lot : [...]. J'ai fait plusieurs essais qui se sont avérés infructueux, et ma recherche en ligne n'a abouti à rien. Merci beaucoup à ceux qui trouveront la réponse.

Réponse 1: TECHDOC: Pour les experts de BCDI: Faire la recherche sur la table notices. Puis, en changement par lot: [...] Passez-moi un courriel si je ne suis pas assez clair (ce qui probable !) Cordialement. »

Nous voyons ici apparaitre l'image du documentaliste expert dans l'utilisation du logiciel professionnel, référent auprès de ces collègues. Cette communication intraprofessionnelle par internet est un lieu d'expertise partagée entre collègues et de débats internes et externes. La photographie de la profession présentée ici est celle de la situation à un moment donné, dans un environnement évoluant en permanence. Notre approche ethnographique de la liste cdidoc, nous a permis de distinguer les thèmes les plus fréquemment évoqués par la communauté professionnelle. Notre position de praticien-chercheur enrichit notre analyse par la connaissance du contexte professionnel et des débats internes à la profession. Le recours à une typologie extérieure au monde de la documentation scolaire favorise une mise à distance par rapport à nos représentations du métier d'enseignant documentaliste.

\section{Conclusion}

L'objet de cet article est de mettre en évidence l'intérêt qu'il y a à concilier approche ethnographique et analyse du discours pour l'étude de notre corpus. L'analyse de discours s'applique aujourd'hui à de nouveaux corpus, comme les discours clos circulant dans des communautés restreintes qui à travers leurs rites langagiers partagent un même territoire. Un élargissement du contexte s'impose à l'analyste, une telle démarche 
suppose en effet de s'efforcer de restituer les connaissances, savoirs, catégories mobilisés par les sujets parlants pour construire le sens. Notre étude limitée ne prétend pas à l'universel, néanmoins le terrain professionnel que nous analysons est porteur d'un potentiel de renseignements pour d'autres situations.

\section{BIBLIOGRAPHIE}

Bakhtine, M., (1984), Esthétique de la création verbale, Paris, Gallimard, 400 p.

Bonnafous, S., (2006), L'analyse du discours, in Olivesi S., dir., Sciences de l'information et de la communication. Objets, savoirs, discipline, Grenoble, PUG, p. 213-228

Canter-Kohn, R., (2001), Les positions enchevêtrées du praticien qui devient chercheur, in Mackiewick M-P. Praticien et chercheur : Parcours dans le champ social. Paris, L'harmattan, p. 15-38

Charaudeau, P. et Montes, R., (2004), La voix cachée du tiers. Des non-dits du discours, Paris, L'Harmattan, 235 p.

D'Almeida, N., (2007), La Société du jugement, Paris, Armand Colin, 225 p.

European Council of Information Associations, (2004), Euroréférentiel I\&D, Volume 1. Compétences et aptitudes des professionnels européens de l'information-documentation. ADBS éditions, $107 \mathrm{p}$.

Kolmayer, E., (2003), Formation et capitalisation des connaissances sur une liste de diffusion professionnelle, in Reseaux 119, p. 176-201

Sarfati, G-E., (2005), Précis de pragmatique. Paris, A. Colin, 127 p.

Wenger, E., (1998), Communities of practice : learning, meaning and identity, Cambridge University Press, $336 \mathrm{p}$.

Winkin, Y., (2001), Anthropologie de la communication. De la théorie au terrain. Bruxelles, De Boeck Université, $239 \mathrm{p}$.

Zarifian, P., (2004), Le modèle de la compétence, Editions Liaisons, 144 p.

\section{RÉSUMÉS}

L'article rend compte d'un travail de recherche sur le fonctionnement d'une communauté de pratiques à travers l'analyse d'une liste de discussion de documentalistes en milieu scolaire. Après une présentation des choix méthodologiques effectués afin de construire l'objet d'étude, sont détaillées les méthodes d'analyse communicationnelle et discursive des échanges. Ces techniques d'enquête sont ensuite interrogées lors d'une étude exploratoire sur les activités professionnelles dominantes présentes au sein du corpus de messages archivés.

The article gives an account of a research work on the operation of a community of practice through the analysis of a list of discussion for librarians in schools. After a display of the methodological choices made to build the object of study, are retailed the methods of analysis 
communicational and discursive of the exchanges. These techniques of survey are then questioned during an exploratory study on the dominant professional activities present within the corpus of archived messages.

INDEX

Mots-clés : liste de discussion, communauté en ligne, documentaliste, savoirs professionnels Keywords : list of discussion, online community, librarian, professional knowledge

\section{AUTEUR}

\section{FLORENCE THIAULT}

Florence Thiault est enseignante en documentation à l'université Rennes 2. Elle prépare une thèse de doctorat à l'université Lille 3 (laboratoire GERiiCO). Ses travaux de recherche portent sur les communautés en ligne et les savoirs professionnels en information-documentation. Adresse électronique : florence.thiault@univ-rennes2.fr 